\section{REPORT INVESTIGATES RISE IN COMPLAINTS AGAINST DOCTORS}

A new report analyses the rise in doctor fitness to practise complaints made by the public to the General Medical Council (GMC).

The GMC commissioned the report from a team at the Collaboration for the Advancement of Medical Education, Research and Assessment (CAMERA) at Plymouth University Peninsula Schools of Medicine and Dentistry.

An increase in complaints has been seen across the UK, which suggests wider social trends rather than localised issues. A large number of complaints did not progress because the issues raised could not be identified, which suggests that the GMC is receiving complaints outside its remit. According to the report, this points towards problems with the wider complaint-handling system and culture.

The authors of the report suggest that media portrayals of both the GMC and the medical profession may influence how and why complaints are made. Negative press coverage may be 'chipping away' at this reputation and resulting in an increased number of people making 'me too' complaints to the GMC.

Niall Dickson, Chief Executive of the GMC, said: 'The challenge for the GMC and other organisations is to make sure that anyone who has a complaint can find their way to the right organisation to deal with it. For the vast majority, that will mean local resolution'.

\section{TRIBUTES TO A LEGEND IN PREVENTIVE DENTISTRY}

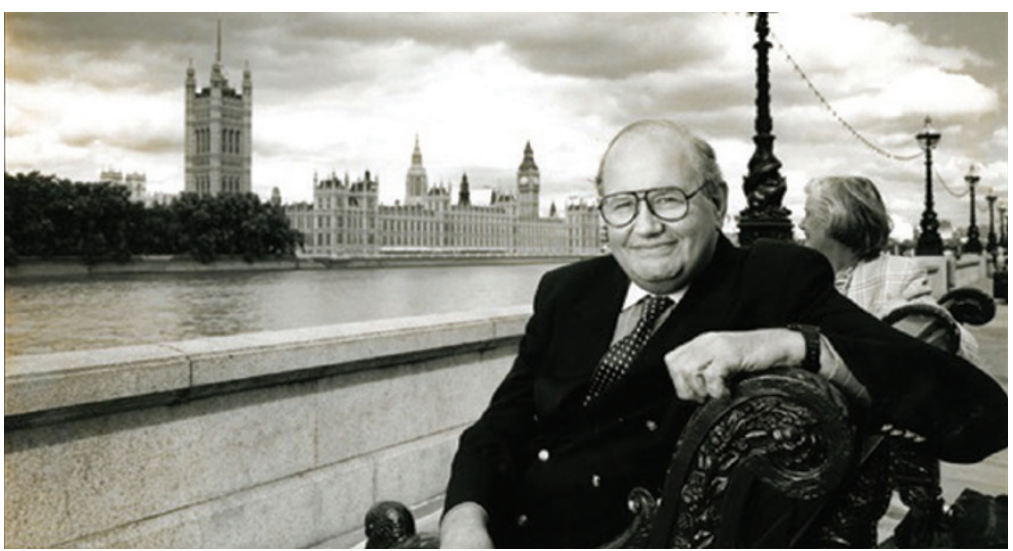

Colin Hall Dexter died on 6 July 2014 and his funeral was held at St Marylebone Parish Church on 25 July at $2 \mathrm{pm}$.

Colin was one of the most influential figures in preventive dentistry and was instrumental in the formation of the British Dental Health Foundation. He was extremely well thought of, respected and loved by many people.

Dr Nigel Carter OBE, Chief Executive of the Foundation, said: 'The passing of Colin Hall Dexter sees the demise of a legend in preventive dentistry and a true friend and lifelong supporter of the British Dental Health Foundation from when he helped to set it up in 1971. It is a great shame that Colin did not live to see some of his ideas on a preventive approach to dentistry being implemented in the
Health Service with the new dental contract. Colin was a pioneer of the preventive approach but had to leave the health service to implement it. He would be delighted that this approach is now being mainstreamed into patient care'

Sally Goss, who was a dental hygienist at Colin's practice in Harley Street, said that 'his support and admiration for the work of dental hygienists knew no bounds. And he was a man so far ahead of his time with his skill in getting the preventive message over to patients.

'Colin could light up a room with his energy and dynamism.'

Dentist Lynn Walters said: 'I remember Colin as a mentor of unbounded enthusiasm in all that he did; a cultured man, and a true friend. It was a privilege to have known him'.

\section{BOOK REVIEW}

\begin{tabular}{|l|l}
\hline $\begin{array}{l}\text { expanded } \\
\text { atraumatic } \\
\text { techniques }\end{array}$ & $\begin{array}{l}\text { A HANDBOOK OF EXPANDED } \\
\text { ATRAUMATIC TECHNIQUES } \\
\text { FOR THE APPREHENSIVE CHILD } \\
\text { DENTAL PATIENT }\end{array}$ \\
& $\begin{array}{l}\text { G. C. Craig, K. R. Powell } \\
\text { Dental Outlook Publications } \\
\text { price } £ 40.50 ; \text { pp } 37 \\
\text { ISBN 9780992311001 }\end{array}$
\end{tabular}

This handbook is intended to expand practitioners' caries management armamentarium for anxious patients. Its primary goal is clear - reduction in the number of children requiring hospital admission for dental extractions under general anaesthetic (GA).

The techniques included have been drawn from the Bourke study, which both authors were involved in. The study ran from 1978-1984 in a remote part of New South Wales, Australia, where caries-risk in children was high and access to GA services was severely limited. In view of this, along with the growing levels of dental anxiety in local children, a three-stage system of acclimatisation was developed.

The authors highlight the need to eliminate all equipment that could be perceived as threatening by children. This would include local anaesthetic apparatus, high-volume suction, matrix bands and probes. It may seem like an impossible task to effectively manage caries without such tools. However, the authors go on to thoroughly describe the values of alternative methods such as silver fluoride, stannous fluoride paste, and ultra low-speed excavation.

The section of this handbook dedicated to the recognition of high-risk sites for caries progression in the primary dentition could be useful as a guide for treatment prioritisation in children. The chapter detailing the use of rotary instruments provides superb suggestions for retentive cavity preparation in primary teeth and is supplemented by a range of photographs, radiographs and diagrams.

UK readers will notice considerable variation from current management principles for grossly carious teeth, retained roots and teeth with acute or chronic infection. Whilst use of a minimally invasive technique is encouraged and forms an essential part of an acclimatisation programme, it must be recognised that there are occasions when it seems unreasonable to go to such lengths to avoid an extraction. Some of the techniques described have perhaps been eclipsed by more modern, evidence-based techniques such as the Hall technique for preformed metal crowns and regular application of topical fluoride vanish.

This is an intriguing read based on a fundamental piece of research that remains useful in controlling caries risk in more isolated communities with severely limited access to dental care. However, in patient populations with reasonable access to modern materials, techniques and facilities, including treatment under general anaesthetic, some of the methods described may not be so relevant.

H. ROGERS 